\title{
Investigation of Penile Conditions by Ultrasound and Contrast-Enhanced Ultrasound - Presentation of Three Clinical Cases
}

\section{Introduction \\ $\nabla$}

Imaging and evaluation of penile conditions are not everyday entities in most radiology departments. Magnetic resonance imaging (MRI), computed tomography (CT), fluoroscopy and ultrasound (US) are the primary imaging techniques of today's practice. The use of contrastenhanced ultrasound (CEUS) in penile conditions is a new entity. We report 3 different cases of penile conditions evaluated by US and contrast-enhanced ultrasound (CEUS) which demonstrate the usefulness of CEUS in the primary evaluation

\section{CASE 1: Penile Tumor}

$\nabla$

A 74-year-old man suffering from prostatic cancer presented with a tumor on the left side of the penis. B-mode US using a linear $2-8 \mathrm{MHz}$ transducer showed a well-delineated heterogeneous tumor with a diameter of $1 \mathrm{~cm}$ close to the tunica albuginea in the left corpus cavernosum ( $\odot$ Fig. 1a). CEUS was performed (1.5 ml of SonoVue ${ }^{\mathrm{TM}}$, Bracco, Milan) and the tumor showed hyperenhancement in the arterial phase ( $\bullet$ Fig. 1b). The conclusion of the US examination was hypervascular tumor, possible metastasis. An autopsy 2 months later confirmed that the tumor in the penis was a metastasis from a neuroendocrine prostatic cancer.

\section{CASE 2: Penile Infection \\ $\nabla$}

A 55-year-old patient presented with a painful mass on the ventral part of the penis. B-mode US showed a well-delineated, homogeneous and hypoechoic lesion measuring $2 \times 3 \mathrm{~cm}(\bullet$ Fig. $2 \mathrm{a}$ ). CEUS $\left(1.5 \mathrm{ml}\right.$ of SonoVue ${ }^{\mathrm{TM}}$, Bracco, Milan) demonstrated an avascular cavity ( $\bullet$ Fig. 2b). The abscess cavity was punctured and pus was aspirated. After repeated flushing with saline, a few drops of Sono-

\section{License terms}

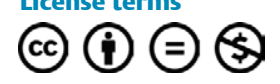

Vue were added to $100 \mathrm{~mL}$ of $0.9 \%$ saline and a few milliliters were injected into the cavity to demonstrate a small communicating fistula between the abscess cavity and the urethra. The patient was treated with antibiotics for a further 5 days, and the fistula was treated in the urology department after remission of the abscess.

\section{Case 3: Penile Trauma $\nabla$}

A 35-year-old man presented with severe penile pain and suspicion of penile fracture after trauma during sexual intercourse. He had a history of penile fracture 2 years previously, treated surgically without any complications. On presentation, the penis was swollen and discolored on the left aspect and with a right deviation. US examination of the penis showed a $1.9 \mathrm{~cm}$ homogeneous mass on the left aspect, in close relation to the left cavernous body, probably a hematoma ( $\odot$ Fig. 3a). Due to difficulty detecting the acute abnormality and the cause, CEUS was performed $\left(1.5 \mathrm{ml}\right.$ of SonoVue ${ }^{\mathrm{TM}}$, Bracco, Milan) which showed an avascular area representing the hematoma and demonstrated a laceration of the left cavernous body ( $\bullet$ Fig. $3 \mathbf{b}$ ). Acute surgery revealed a $5 \mathrm{~mm}$ lesion in the tunica albuginea on the left cavernous body. The patient was discharged without any complications after 2 days.

\section{Discussion \\ $\nabla$}

This is the first case report presenting the use of CEUS in patients with penile abscess, fracture or metastasis. CEUS adds to the information presented on B-mode scanning and in some cases the technique makes a total bedside evaluation possible. Penile metastases are very rare and less than 450 cases have been reported in the literature (Chaux A et al. Int J Surg Pathol. 2011; 19: 597-606). Penile metastases are usually seen in the erectile tissue, whereas primary penile cancers are more common in the prepuce or on the penile glans. MRI is reported to have a higher ac- curacy compared to US (B-mode) and CT regarding the differential diagnosis in cases where clinical suspicion is established (Chaux A et al. Int J Surg Pathol. 2011; 19: 597-606). CEUS and fine needle aspiration (FNAC) would be a quick and low-cost option compared to $\mathrm{CT}$ and MRI. Primary symptoms of metastases are a round-shaped filling in the penile shaft or priapism. Additional symptoms may be dysuria, hematuria and pain. The spread is probably primarily retrograde from the venous plexus of the pelvis to the penile dorsal venous system, and the majority of penile metastases are from the prostate, bladder and the lower gastrointestinal tract. Penile metastases are most often seen in disseminated disease and correlated to a poor prognosis.

A penile abscess is a rare urologic abnormality. Primary symptoms are penile swelling and pain. Pyrexia, dysuria or urethral discharge may be seen. The majority of penile abscesses are located in the corpus cavernosum and related to an intracavernosal injection, perianal/ perineal/intraabdominal abscess, penile trauma and instrumentation (Dugdale CM et al. Curr Urol 2013; 7: 51-56). Immunosuppression, particularly diabetes mellitus, predisposes to penile abscess. The clinical diagnosis is usually obvious, but in difficult cases with widespread infection in the perineal area, further imaging with CT or MRI is helpful. Most reported cases are treated by surgery, but often with postoperative complications, such as penile curvature and erectile dysfunction. CEUS is effective for the detection and evaluation of a cavity, and in this case with localized infection it demonstrated a communicating fistula to the urethra, the potential reason for abscess recurrence (Piscaglia Fet al. Ultraschall Med. 2012; 33: 33-59). The patient was diagnosed, treated and evaluated in one clinical setting which underlines some of the advantages of US and CEUS imaging. Penile fracture is caused by rupture of the cavernosal tunica albuginea and may be associated with subcutaneous hematoma and lesions of the urethra or the corpus spongiousm, arising in $10-20 \%$ of cases (Koifman KL et al. Urology. 2010; 76: 1488-1492). A subcutaneous hematoma without rupture of the tunica albuginea does not require surgical intervention. In penile fracture, acute surgical intervention with closure of the tunica albuginea is recommended (Koifman KL et al. Urology. 2010; 76: 1488-1492). The rate of postoperative complications has been re- 

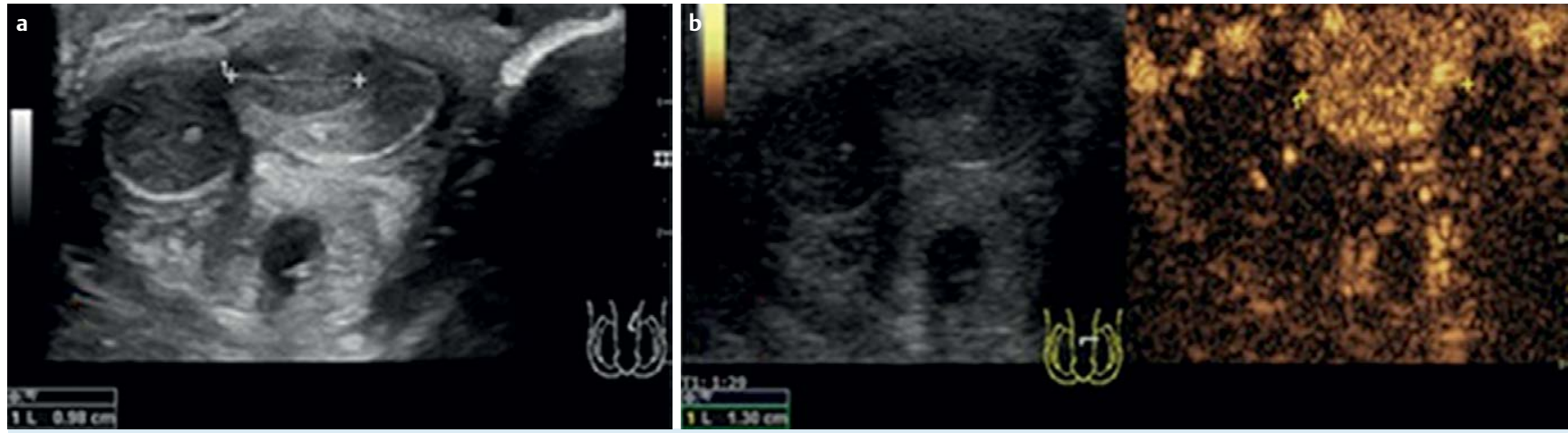

Fig. 1 a Transverse B-mode image (using a linear 2-8MHz tranducer) showing the tumor in the left corpus cavernosum (between cursors). b Transverse CEUS dual mode arterial phase image showing the hypervascular tumor in the left copus cavernosum (between cursors).
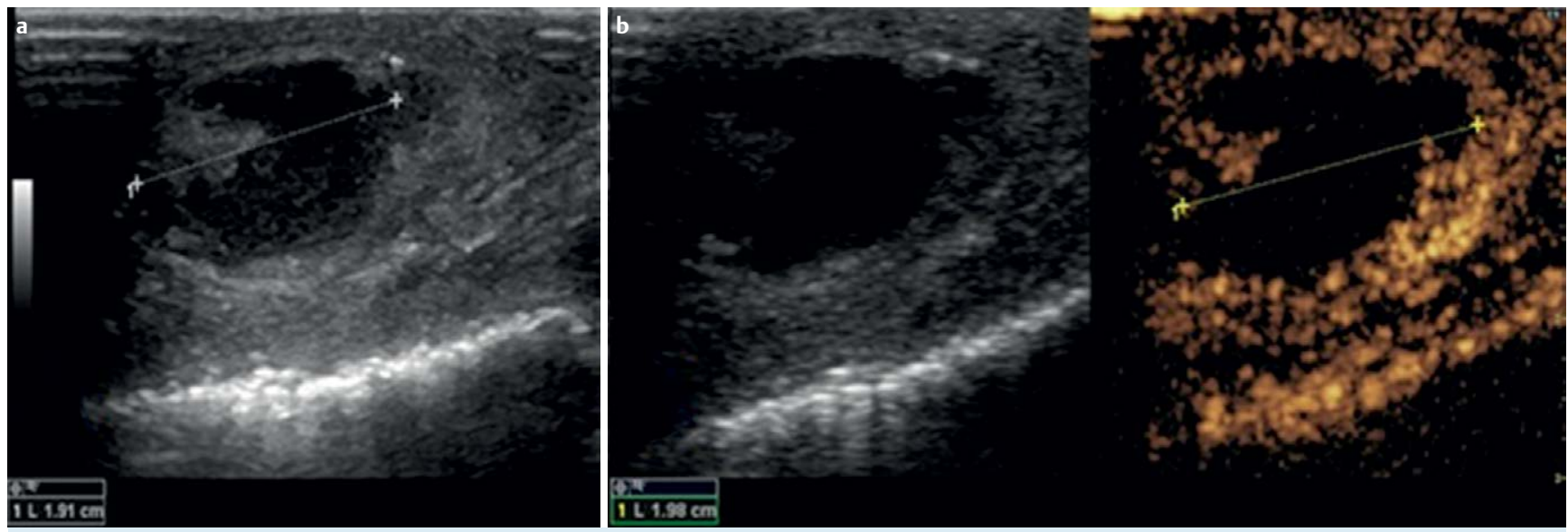

Fig. 2 a B-mode image (using a linear 2-8MHz tranducer) showing the hypo-echoic cavity in the corpus spongiosum. $\mathbf{b}$ Following the iv. administration of contrast, the abscess cavity is well demonstrated in the CEUS dual mode.
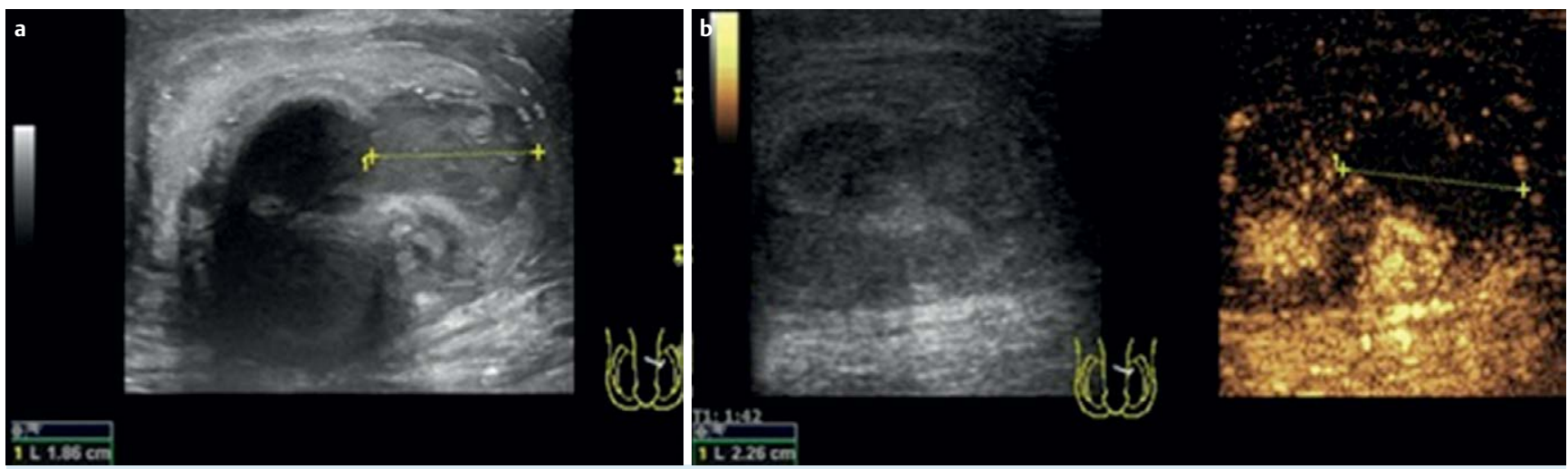

Fig. 3 a Transverse B-mode image (using a linear $2-8 \mathrm{MHz}$ tranducer) showing the haematoma (between cursors) in close relation to the left cavernous body. $\mathbf{b}$ Late phase transverse CEUS dual mode showing the avascular area representing the haematoma.

ported as $9 \%$, including erectile dysfunction in $1.3 \%$ (Haas CA et al. World J Urol 1999; 17: 101-106). Nonsurgical treatment of penile facture is related to an increased rate of complications such as erectile dysfunction and penile curvature. MRI or US may identify the laceration in the tunica albuginea in atypical cases or confirm that the tunica is intact.
US and CEUS using high-frequency linear transducers for the evaluation of penile conditions are technically feasible and are useful imaging techniques.

J. F. Topsøe, D. Dencker; Denmark 